\title{
RESEARCH
}

Open Access

\section{Determinants of low birth weight deliveries at five referral hospitals in Western Area Urban district, Sierra Leone}

David Kabba Kargbo 1,2,3, Kofi Nyarko3 ${ }^{3}$, Samuel Sackey ${ }^{1,2}$, Adolphina Addo-Lartey ${ }^{2}$, Ernest Kenu ${ }^{1,2}$ and Francis Anto ${ }^{2^{*}}$ (D)

\begin{abstract}
Background: Low birth weight (LBW) contributes significantly to infant and child mortality. Each year, about 20 1 million deliveries are LBW with $96.5 \%$ occurring in developing countries. Whiles the incidence of LBW is reducing in other districts of Sierra Leone, it has been reported to be increasing in the Western Area Urban district. Determining the risk factors in a specific geographic area is important for identifying mothers at risk and thereby for planning and taking appropriate action. The current study sought to identify factors associated with LBW deliveries in the Western Area Urban district of Sierra Leone.
\end{abstract}

Methods: A hospital-based unmatched 1:2 case-control study was conducted among mothers who delivered live singleton babies from November, 2019 to February, 2020 in five referral health facilities. Mothers were conveniently sampled and sequentially enrolled into the study after delivery. Their antenatal care cards were reviewed and a pretested questionnaire administered to the mothers. Data analysis was done using Stata 15.0 and association between maternal socio-demographic, socio-economic, obstetric and lifestyle factors and LBW assessed using bivariable and multivariable logistic regression analyses.

Results: A total of 438 mothers (146 cases and 292 controls), mean age: $24.2( \pm 5.8)$ and 26.1 ( \pm 5.5$)$ years for cases and controls respectively participated in the study. Multivariable analysis revealed that being unemployed $(A \circ R=$ $2.52,95 \% \mathrm{Cl} 1.16-5.49, p=0.020$ ), having anaemia during pregnancy (AoR $=3.88,95 \% \mathrm{Cl} 1.90-7.90, p<0.001$ ), having less than 2 years inter-pregnancy interval ( $\mathrm{AoR}=2.53,95 \% \mathrm{Cl} 1.11-5.73, p=0.026)$, and smoking cigarettes during pregnancy (AoR $=4.36,95 \% \mathrm{Cl} 1.94-9.80, p<0.001$ ) were significantly associated with having LBW babies.

Conclusion: Factors associated with LBW identified were unemployment, anaemia during pregnancy, $<2$ years inter-pregnancy interval and cigarette smoking during pregnancy. Health care providers should screen and sensitize mothers on the risk factors of LBW during antenatal sessions.

Keywords: Low birth weight, Case-control, Referral hospitals, Western area urban, Sierra Leone

\footnotetext{
*Correspondence: fanto@ug.edu.gh

${ }^{2}$ Department of Epidemiology and Disease Control, School of Public Health, University of Ghana, Accra, Ghana

Full list of author information is available at the end of the article
}

(c) The Author(s). 2021 Open Access This article is licensed under a Creative Commons Attribution 4.0 International License, which permits use, sharing, adaptation, distribution and reproduction in any medium or format, as long as you give appropriate credit to the original author(s) and the source, provide a link to the Creative Commons licence, and indicate if changes were made. The images or other third party material in this article are included in the article's Creative Commons licence, unless indicated otherwise in a credit line to the material. If material is not included in the article's Creative Commons licence and your intended use is not permitted by statutory regulation or exceeds the permitted use, you will need to obtain permission directly from the copyright holder. To view a copy of this licence, visit http://creativecommons.org/licenses/by/4.0/ The Creative Commons Public Domain Dedication waiver (http://creativecommons.org/publicdomain/zero/1.0/) applies to the data made available in this article, unless otherwise stated in a credit line to the data. 


\section{Background}

Low birth weight (LBW) is of major public health concern especially in low and middle-income countries [1]. It contributes significantly to the burden of childhood diseases and increases mortality and disability in neonates and infants [2]. LBW is the weight of a newborn measuring less than $2.5 \mathrm{~kg}$ and is measured immediately after birth [3]. According to Agbor et al. however, it has become increasingly evident that the traditional cut-off value of $2.5 \mathrm{~kg}$ advocated by WHO to define LBW may not be generalizable to all settings, as variations have been reported from rural and sub-urban settings of even the same country [4]. In their study in rural Cameroon a cut-off value of $2.7 \mathrm{~kg}$ was arrived at which supported an earlier report from a sub-urban setting by Nim et al. [5] that reported a value of $2.6 \mathrm{~kg}$ as cut-off for LBW.

Globally, 15 to $20 \%$ of total births are LBW representing about 20 million births a year [6]. About $96.5 \%$ of LBW deliveries in 2019 occurred in developing countries; accounting for $60-80 \%$ of neonatal deaths [7]. The prevalence of LBW varies from region to region and within countries; with Asia accounting for 28\%, Africa 13\% and Latin America 9\% [6].

In Sierra Leone, the pattern of LBW deliveries is unstable. The 2008 and 2013 Sierra Leone Demographic and Health Survey (SLDHS) showed that nationwide, LBW deliveries decreased from $11.0 \%$ in 2008 to $7.1 \%$ in 2013. However, it rather increased in the Western Area Urban district from 9.5 to $17.5 \%$ over the same period $[8,9]$.

Newborns with LBW are at greater risk of neonatal mortality compared to normal weight babies [7]. Management of LBW deliveries increases health care costs due to long periods of hospitalization. It is estimated that care for extremely LBW babies is about six times more expensive compared to normal weight babies [10]. Evidence also exists that LBW increases the likelihood of developing non-communicable diseases like diabetes and cardiovascular diseases in adulthood $[10,11]$. LBW has short term consequences, including respiratory distress, infections and hydrocephalus as well as long-term consequences, including coronary heart disease, diabetes, hypertension, and impaired cognitive function [12].

WHO has made a number of recommendations aimed at reducing the number of LBW infants by $30 \%$ between 2012 and 2025. These recommendations include, adequate nutrition for adolescent girls, promotion of smoking cessation during and after pregnancy, community-based packages of care to improve linkage and referral for facility births. Others are, intermittent iron and folic acid supplements for women of reproductive age and adolescent girls, in settings where the prevalence of anaemia is $20 \%$ or higher and prevention of malaria during pregnancy [13]. All these are implementable at the community level to reduce low birthweight deliveries and therefore neonatal mortality.

Several factors including early initiation of labour, mother's lifestyle (e.g. smoking and drinking alcohol), multiple pregnancies, maternal age $<15$ years or $>35$ years, poverty, infections and chronic illnesses including diabetes and hypertension have been reported to contribute to the delivery of LBW babies [11]. The purpose of the current study was to identify factors contributing to LBW in Sierra Leone to allow for the development of sustainable preventive measures in order to reduce the cost to the health sector. This study, therefore, assessed the socio-demographic, obstetric, maternal, and lifestyle factors associated with LBW babies delivered in five referral hospitals in WAU, Sierra Leone.

\section{Methods \\ Study design}

A hospital-based unmatched case-control study was undertaken in five referral hospitals in the Western Area Urban district of Sierra Leone from November, 2019 to February, 2020 among mothers who delivered singleton live babies. For each mother who delivered a LBW baby, two mothers who subsequently delivered normal weight babies in the same hospital were selected as controls.

\section{Study setting}

The study was conducted in five-referral hospitals (Government and Non-governmental organization (NGO) supported) offering free maternal services: Princess Christian Maternity Hospital (PCMH), Lumley Government Hospital, King Harman Road Government Hospital, 34 Military hospital, and Aberdeen Women's Centre (NGO). All these facilities were located within the capital city, Freetown. In 2019, the WAU district had a projected population of 1.2 million. The district is divided into 20 zones and has five referral hospitals and 71 peripheral health units (PHUs). The district recorded 6112 deliveries in health facilities in 2019.

\section{Study variables}

The independent variables included socio-demographic factors (age of mother, educational level, ethnicity, marital status and religion), socio-economic factors, (family income level and occupation), obstetric factors (maternal BMI, weight, height, parity, gravidity, abortion, ANC visits, gestational age, birth interval and anaemia during pregnancy), maternal health and lifestyle factors (diseases like hypertension, diabetes, heart disease, HIV, syphilis and malaria, alcohol intake, smoking and use of herbal medication). The dependent variable was low birth weight. 


\section{Study participants and eligibility criteria}

Mothers who delivered live singleton babies in the five referral hospitals during the study period were eligible to participate in the study. The weight of every newborn was measured within an hour of birth. Mothers with unknown last normal menstrual period, caesarean section birth, congenital deliveries, stillbirths and seriously ill mothers were excluded from the study.

\section{Case definition}

Mothers who delivered babies weighing less than $2.5 \mathrm{~kg}$ and consented to take part in the study were enrolled as cases.

\section{Control definition}

Mothers with newborns weighing $2.5 \mathrm{~kg}$ and above were enrolled as controls.

\section{Sample size determination}

An online OpenEpi software, version three for unmatched case-control studies was used to estimate the required sample size. A minimum detectable odds ratio of two and a percentage control group (birth spacing less than 2 years) exposed (67.6\%) from a study conducted in Ethiopia was used [14]. Birth spacing was selected as an independent variable as it gave a higher sample size. A case to control ratio of 1:2 with a $95 \%$ confidence level and $80 \%$ power was used. The required sample size estimated was 438 (146 cases and 292 controls).

\section{Sampling procedure}

A total of 251 singleton live LBW babies were delivered in the five hospitals from November, 2018 to February, 2019 as follows: PCMH:138; King Harman Road Government Hospital: 3; Lumley Government Hospital: 15; 34 Military hospital: 8; and Aberdeen Women's Centre: 87. The sample size was distributed proportionately among the five facilities based on the November, 2018 to February, 2019 singleton LBW deliveries per facility. The convenience sampling method was used, during which mothers were enrolled sequentially as they delivered until the required sample size for the facility was attained. For each mother who delivered a LBW baby, two mothers who subsequently delivered normal weight babies in the same hospital were selected as controls. In a situation where a case or control declined consent to be part of the study, the next eligible mother was selected as a replacement.

\section{Data collection tools and procedure}

A questionnaire was developed specifically for this study based on the objectives of the study and used for data collection from mothers who met the inclusion criteria of the study. The questionnaire was in three sections: socio-demographic and socio-economic, obstetric, and maternal health and lifestyle factors. The ANC cards of the mothers were reviewed and data on obstetric and maternal health factors extracted. Fifteen trained research assistants (three per hospital and one per shift) collected the data through face-to-face interviews using the common language used in the study area, 'Creole'. Midwives took the weights of newborn babies within one hour after delivery using calibrated Seca 354 weighing scales (seca gmbh \& co.kg 22,089 Hamburg, Germany). These weights, were extracted from the ANC cards of the mothers. The research assistants administered the questionnaires to mothers within $24 \mathrm{~h}$ postdelivery in a separate office within the ward to maintain privacy. Data were collected simultaneously in the five hospitals until the required sample size was obtained.

\section{Quality control}

The questionnaire was developed specifically for this study based on the objectives of the study, known variables from literature and the research experience of the authors. The questionnaire was pre-tested in three Government and NGO supported hospitals in the WAU district with similar service characteristics using $2 \%$ (three cases and six controls) of the sample size (cases). Findings of the pilot study were used to finalize the questionnaire. The data collected were checked daily for completeness and accuracy by a supervisor. The data were cleaned and entered in SPSS version 22 software and password protected.

\section{Data processing and analysis}

Statistical analysis was done using Stata 15.0 (Stata Corp, College Station, TX USA). A histogram-normal curve was used to check the normality of continuous variables. Descriptive analysis i.e. summary statistics, mean (SD) and proportions were estimated, and inferential analysis using chi-square and logistic regression (bivariable and multivariable) to determine association between LBW and the independent variables. A stepwise backward elimination method with a restricted alpha level of $0.1(10 \%)$ was conducted and the post estimation command ("testparm i.variablename") used to determine variables that met the criteria for the multivariable logistic model. Odds ratio (OR) with $95 \% \mathrm{CI}$ was computed and statistical significance was determined at $p$-value $<0.05$.

\section{Ethical consideration}

Ethical clearance was obtained from the Sierra Leone Ethics and Scientific Review Committee (SLESRC). Permission was also obtained from the Chief Medical Officer, Ministry of Health and Sanitation (MoHS), and the hospital administration. The possible risk of the study (time) was explained to the mothers and their worries 
addressed. Written informed consent was obtained from each mother or her parent or guardian (mothers less than 18 years old) before data collection. The information collected was treated as confidential and codes were used to identify participants and not names.

\section{Results}

\section{Socio-demographic characteristics of the mothers}

A total of 438 mothers (146 cases and 292 controls) were enrolled into the study from the five-referral hospitals. They were aged 14-39 years, with mean age of 24.2 (SD: 5.8) years for cases and 26.1 (SD: 5.5) years for controls $(p=0.685)$. One hundred and sixty-two of the mothers (36.9\%) had no formal education, $25.8 \%$ (113) were unemployed and 29.7\% (130) were not married (Table 1).

Bivariable logistic regression analysis of the sociodemographic characteristics of the mothers revealed statistical differences between cases and controls in terms of age $(\mathrm{CoR}=3.08,95 \% \mathrm{CI} 1.60-5.92, p=0.001)$, employment $(\mathrm{CoR}=4.06,95 \% \mathrm{CI} 2.54-6.47, p<0.001)$, and marital status $(\mathrm{CoR}=1.94,95 \% \mathrm{CI} 1.25-3.01, p=$ 0.003) (Table 2).

\section{Obstetric characteristics of the mothers}

Concerning the obstetric profile, shorter mothers were observed to be higher in cases than controls $(32.2 \%$ vs $7.8 \%, \mathrm{p}=0.001)$. A higher proportion of case mothers made less than four ANC visits compared to control mothers $(41.1 \%$ vs $20.5 \%, p<0.001)$. The mothers who had anaemia during pregnancy were significantly higher among cases than controls $(56.2 \%$ vs $21.9 \%, p<0.001)$. Mothers who had less than 2 years inter-pregnancy interval were observed to be higher among cases than controls $(29.3 \%$ vs $15.4 \%, p=0.008)$. There was no significant difference in the proportion of mothers who had a history of abortion between cases and controls $(28.8 \%$ vs 25.0, $p=0.398$ ). Table 3 displays the obstetric factors found to be associated with delivery of low birth weight babies after bivariable analysis. These factors included, maternal height $(\mathrm{CoR}=2.19,95 \%$ CI 1.34-3.55, $p=$ $0.001)$, gravidity $(\mathrm{CoR}=1.81,95 \%$ CI $1.18-2.76, p=$ $0.003)$, number of ANC visits made before delivery $(\mathrm{CoR}=2.69,95 \%$ CI 1.70-4.26, $p<0.001)$, and gestational age at delivery $(\mathrm{CoR}=12.58,95 \%$ CI $1.66-94.84$, $p=0.014)$. Other factors are anaemia during pregnancy

Table 1 Socio-demographic characteristics of the mothers, WAU, 2020

\begin{tabular}{|c|c|c|c|}
\hline \multirow[t]{2}{*}{ Characteristics } & Cases (\%) & Controls (\%) & Total (\%) \\
\hline & $n=146$ & $n=292$ & 438 \\
\hline \multicolumn{4}{|l|}{ Mother's age (years) } \\
\hline$<20$ & $36(24.6)$ & $30(10.3)$ & $66(15.1)$ \\
\hline $20-29$ & $82(56.2)$ & $190(65.1)$ & $272(62.1)$ \\
\hline$\geq 30$ & $28(19.2)$ & $72(24.6)$ & $100(22.8)$ \\
\hline \multicolumn{4}{|l|}{ Education } \\
\hline No formal education & $64(43.8)$ & $98(33.6)$ & $162(36.9)$ \\
\hline Primary & $23(15.8)$ & $40(13.7)$ & $63(14.4)$ \\
\hline Secondary & $42(28.8)$ & $113(38.7)$ & $155(35.4)$ \\
\hline Tertiary & $17(11.6)$ & $41(14.0)$ & $58(13.2)$ \\
\hline \multicolumn{4}{|l|}{ Employment status } \\
\hline Unemployed & $63(43.1)$ & $50(17.1)$ & $113(25.8)$ \\
\hline Student & $20(13.7)$ & $39(13.4)$ & $59(13.5)$ \\
\hline Employed & $63(43.2)$ & $203(69.5)$ & $266(60.7)$ \\
\hline \multicolumn{4}{|l|}{ Mother/household monthly income } \\
\hline$<$ Le 500,000 & $121(82.9)$ & $226(77.4)$ & $347(79.2)$ \\
\hline$\geq$ Le 500,000 & $25(17.1)$ & $66(22.6)$ & $91(20.8)$ \\
\hline \multicolumn{4}{|l|}{ Marital status } \\
\hline Single & $55(37.7)$ & $75(25.7)$ & $130(29.7)$ \\
\hline Cohabiting/married husband unemployed & $15(10.3)$ & $15(5.1)$ & $30(6.8)$ \\
\hline Cohabiting/married husband employed & $76(52.0)$ & $202(69.2)$ & $278(63.5)$ \\
\hline \multicolumn{4}{|l|}{ Religious affiliation } \\
\hline Christian & $60(41.1)$ & $110(37.7)$ & $170(38.8)$ \\
\hline Muslim & $86(58.9)$ & $182(62.3)$ & $268(61.2)$ \\
\hline
\end{tabular}


Table 2 Comparison of socio-demographic and socio-economic characteristics of mothers of cases and controls, WAU, 2020

\begin{tabular}{|c|c|c|c|c|}
\hline Characteristics & Cases $(n=146)$ & Controls $(n=292)$ & $\begin{array}{l}\text { Crude OR } \\
(95 \% \mathrm{Cl})\end{array}$ & $p$-value \\
\hline \multicolumn{5}{|l|}{ Mother's age (years) } \\
\hline$<20$ & 36 & 30 & $3.08(1.60-5.92)$ & $0.001^{*}$ \\
\hline $20-29$ & 82 & 190 & $1.10(0.66-1.84)$ & 0.688 \\
\hline$\geq 30$ & 28 & 72 & 1 & \\
\hline \multicolumn{5}{|l|}{ Mother's highest education } \\
\hline No formal education & 64 & 98 & $1.57(0.82-3.00)$ & 0.169 \\
\hline Primary & 23 & 40 & $1.38(0.64-2.97)$ & 0.401 \\
\hline Secondary/Tec-Voc & 42 & 113 & $0.89(0.45-1.74)$ & 0.748 \\
\hline Tertiary & 17 & 41 & 1 & \\
\hline \multicolumn{5}{|l|}{ Mother's employment } \\
\hline Unemployed & 63 & 50 & $4.06(2.54-6.47)$ & $<0.001^{*}$ \\
\hline Student & 20 & 39 & $1.65(0.89-3.03)$ & 0.106 \\
\hline Employed/self-employed & 63 & 203 & 1 & \\
\hline \multicolumn{5}{|l|}{ Mother/household monthly income } \\
\hline$<$ Le 500,000 & 121 & 226 & $1.41(0.83-2.46)$ & 0.182 \\
\hline$\geq$ Le 500,000 & 25 & 66 & 1 & \\
\hline \multicolumn{5}{|l|}{ Marital status } \\
\hline Single & 55 & 75 & $1.94(1.25-3.01)$ & $0.003^{*}$ \\
\hline Cohabiting/married husband unemployed & 15 & 15 & $2.65(1.23-5.69)$ & $0.012^{*}$ \\
\hline Cohabiting/married husband employed & 76 & 202 & 1 & \\
\hline \multicolumn{5}{|l|}{ Religious affiliation } \\
\hline Christian & 60 & 110 & $1.15(0.75-1.76)$ & 0.488 \\
\hline Muslim & 86 & 182 & 1 & \\
\hline
\end{tabular}

$1=$ Reference group; ${ }^{*} \mathrm{p}$-value $<0.05$ is statistically significant; Crude odd ratio (OR), Cl (confidence interval)

$(\mathrm{CoR}=4.56,95 \%$ CI 2.90-7.16, $p<0.001)$, and birth spacing $(\mathrm{CoR}=2.26,95 \%$ CI $1.15-4.39, p=0.008)$.

\section{Maternal health and lifestyle factors}

Hypertension during pregnancy was higher in case mothers than control mothers $(32.2 \%$ vs $9.6 \%$, $p<0.001)$. Mothers with malaria infection during pregnancy were more seen in cases compared to the controls (72.6\% vs $46.9 \%, p<0.001)$. Mothers who smoked cigarette during pregnancy were higher in cases compared to the control (35.6\% vs 9.9\%, $p<0.001$ ). A higher proportion of mothers who took herbal medicine during pregnancy were significantly higher in cases than controls $(54.8 \%$ vs $26.7 \%, p<0.001)$. Bivariable analysis showed statistically significant differences between cases and controls in terms of maternal health factors: hypertension status (CoR $=4.47,95 \%$ CI 2.57-7.83, $p<0.001)$, HIV status ( $\mathrm{CoR}=3.31,95 \%$ CI 1.14-10.29, $p=0.015)$, syphilis infection $(\mathrm{CoR}=3.87,95 \%$ CI 2.01-7.55, $p<0.001)$, malaria during pregnancy $(\mathrm{CoR}=2.99,95 \%$ CI 1.91-4.73, $p<0.001)$, duration of iron and folic acid use $(\mathrm{CoR}=3.55,95 \%$ CI 1.92-6.60, $p<0.001)$. The lifestyle factors associated with delivery of LBW babies were: smoking or living with a partner who smokes $(\mathrm{CoR}=5.01,95 \%$ CI 2.92-8.67, $p<0.001)$ and $(\mathrm{CoR}=$ $2.31,95 \%$ CI $1.49-3.56, p<0.001)$ respectively, and taking herbal medicines during pregnancy $(\mathrm{CoR}=3.32$, $95 \%$ CI 2.14-5.15, $p<0.001$ ) (Table 4).

\section{Determinates of delivery of LBW babies}

After multivariable logistic regression analysis and controlling for possible confounders, factors found to be significantly associated with delivery of LBW babies were: unemployment $\quad(\mathrm{AoR}=2.52,95 \%$ CI 1.16-5.49, $p=$ 0.020 ) (Table 5), anaemia during pregnancy (AoR $=3.88$, 95\% CI 1.90-7.90, $p<0.001$ ), having less than two years inter-pregnancy interval $(\mathrm{AoR}=2.53,95 \%$ CI 1.11-5.73, $p=0.026)$ and cigarette smoking during pregnancy (AoR $=4.36,95 \%$ CI 1.94-9.80, $p<0.001$ ) (Table 6). Even though bivariable analysis showed statistically significant differences between cases and controls in terms of maternal age, marital status, height, gravidity and gestational age at delivery, these factors were no more significantly associated with delivery of LBW babies after 
Table 3 Obstetric factors associated with delivery of low birth weight babies, WAU, 2020

\begin{tabular}{|c|c|c|c|c|}
\hline Characteristics & Cases $(n=146)$ & Controls $(n=292)$ & Crude OR $(95 \% \mathrm{Cl})$ & $p$-value \\
\hline \multicolumn{5}{|l|}{ Mother's weight (kg) } \\
\hline$<50$ & $4(2.7)$ & $9(3.1)$ & $0.88(0.19-3.24)$ & 0.842 \\
\hline$\geq 50$ & $142(97.3)$ & $283(96.9)$ & 1 & \\
\hline \multicolumn{5}{|l|}{ Mother's height (m) } \\
\hline$<1.5$ (short) & $47(32.2)$ & $52(17.8)$ & $2.19(1.34-3.55)$ & $0.001^{*}$ \\
\hline$\geq 1.5$ (normal) & $99(67.8)$ & $240(82.2)$ & 1 & \\
\hline \multicolumn{5}{|l|}{ Mother's BMI $\left(\mathrm{kg} / \mathrm{m}^{2}\right)$} \\
\hline < 18.5 (underweight) & $4(2.7)$ & $9(3.1)$ & $1.26(0.35-4.45)$ & 0.715 \\
\hline 18.5-24.9 (normal) & $35(23.9)$ & $48(16.4)$ & $2.07(1.11-3.87$ & 0.022 \\
\hline 25-29.9 (overweight) & $81(55.5)$ & $161(55.1)$ & $1.43(0.85-2.40)$ & 0.176 \\
\hline$\geq 30$ (obese) & $26(17.8)$ & $74(25.3)$ & 1 & \\
\hline \multicolumn{5}{|l|}{ Parity } \\
\hline Primiparous & $83(56.8)$ & $148(50.7)$ & $1.28(0.84-1.95)$ & 0.223 \\
\hline Multiparous & $63(43.2)$ & $144(49.3)$ & 1 & \\
\hline \multicolumn{5}{|l|}{ Gravidity } \\
\hline Primigravida & $80(54.8)$ & $117(40.1)$ & $1.81(1.18-2.76)$ & $0.003^{*}$ \\
\hline Multigravida & $66(45.2)$ & $175(59.9)$ & 1 & \\
\hline \multicolumn{5}{|l|}{ ANC Visits } \\
\hline$<4$ times & $60(41.1)$ & $60(20.5)$ & $2.69(1.70-4.26)$ & $<0.001^{*}$ \\
\hline$\geq 4$ times & $86(58.9)$ & $232(79.5)$ & 1 & \\
\hline \multicolumn{5}{|l|}{ Gestational age (weeks) } \\
\hline Preterm $(<37)$ & $134(91.8)$ & $213(72.9)$ & $12.58(1.66-94.84)$ & $0.014^{*}$ \\
\hline Term (37-40) & $11(7.5)$ & $59(20.2)$ & $3.72(0.45-30.72)$ & 0.221 \\
\hline Post term $(>40)$ & $1(0.7)$ & $20(6.9)$ & 1 & \\
\hline \multicolumn{5}{|l|}{ Anaemia during pregnancy } \\
\hline Anaemic $(\mathrm{Hb}<11.0 \mathrm{~g} / \mathrm{dl})$ & $82(56.2)$ & $64(21.9)$ & $4.56(2.90-7.16)$ & $<0.001^{*}$ \\
\hline Not anaemic $(\mathrm{Hb} \geq 11.0 \mathrm{~g} / \mathrm{dl})$ & $64(43.8)$ & $228(78.1)$ & 1 & \\
\hline \multicolumn{5}{|l|}{ Birth spacing } \\
\hline$<2$ years & $24(29.3)$ & $29(15.4)$ & $2.26(1.15-4.39)$ & $0.008^{*}$ \\
\hline$\geq 2$ years & $58(70.7)$ & $159(84.6)$ & 1 & \\
\hline \multicolumn{5}{|l|}{ Previous abortion } \\
\hline Ever had & $42(28.8)$ & $73(25.0)$ & $1.21(0.75-1.93)$ & 0.398 \\
\hline Never had & $104(71.2)$ & $219(75.0)$ & 1 & \\
\hline
\end{tabular}

$1=$ Reference group; ${ }^{*} p$-value $<0.05$ is statistically significant

multivariable logistic regression analysis (Table 5). Similarly, factors such as hypertension status, HIV status, syphilis infection, malaria during pregnancy, duration of iron and folic acid use, were no more significantly associated with delivery of LBW babies after multivariable logistic regression analysis (Table 6).

\section{Discussion}

An unmatched case-control study was conducted among women who delivered in five referral hospitals in the Western Area Urban district of Sierra Leone to determine factors associated with delivery of low birth weight (LBW) babies. This study has identified some socio-demographic, socio-economic, obstetric and lifestyle-related factors that are associated with the delivery of LBW babies in the study area.

Earlier studies from across Africa have reported some association between the delivery of LBW babies and several maternal socio-demographic, socio-economic, obstetric and medical factors [15-17]. In our current study, mothers who were unemployed were found to have a two-fold odds of giving birth to a LBW baby. This is 
Table 4 Maternal health and lifestyle factors associated with delivery of LBW babies, WAU, 2020

\begin{tabular}{|c|c|c|c|c|}
\hline Determinants & $\begin{array}{l}\text { Cases } \\
\mathrm{n}(\%)\end{array}$ & $\begin{array}{l}\text { Controls } \\
\mathrm{n}(\%)\end{array}$ & $\begin{array}{l}\text { Crude OR } \\
(95 \% \mathrm{Cl})\end{array}$ & $P$-value \\
\hline \multicolumn{5}{|l|}{ Diabetes } \\
\hline Diabetic & $3(2.1)$ & $18(6.2)$ & $0.31(0.05-1.12)$ & 0.057 \\
\hline Not Diabetic & $143(97.9)$ & $274(93.8)$ & 1 & \\
\hline \multicolumn{5}{|l|}{ Hypertension } \\
\hline Hypertensive & $47(32.2)$ & $28(9.6)$ & $4.47(2.57-7.83)$ & $<0.001^{*}$ \\
\hline Not hypertensive & $99(67.8)$ & $264(90.4)$ & 1 & \\
\hline \multicolumn{5}{|l|}{ Heart disease } \\
\hline Heart disease & $3(2.1)$ & $5(1.7)$ & $1.20(0.18-6.28)$ & 0.800 \\
\hline No Heart disease & $143(97.9)$ & $287(98.3)$ & 1 & \\
\hline \multicolumn{5}{|l|}{ HIV } \\
\hline Positive & $11(7.5)$ & $7(2.4)$ & $3.31(1.14-10.29)$ & $0.010^{*}$ \\
\hline Negative & $135(92.5)$ & 285 (97.6) & 1 & \\
\hline \multicolumn{5}{|l|}{ Syphilis } \\
\hline Positive & $31(21.2)$ & $19(6.5)$ & $3.87(2.01-7.55)$ & $<0.001^{*}$ \\
\hline Negative & $115(78.8)$ & $273(93.5)$ & 1 & \\
\hline \multicolumn{5}{|l|}{ Malaria } \\
\hline Positive & $106(72.6)$ & $137(46.9)$ & $2.99(1.91-4.73)$ & $<0.001^{*}$ \\
\hline Negative & $40(27.4)$ & $155(53.1)$ & 1 & \\
\hline \multicolumn{5}{|l|}{ Iron \& folic acid used } \\
\hline$<3$ months & $34(23.3)$ & $23(7.9)$ & $3.55(1.92-6.60)$ & $<0.001^{*}$ \\
\hline$\geq 3$ months & $112(76.7)$ & $269(92.1)$ & 1 & \\
\hline \multicolumn{5}{|l|}{ Alcohol use } \\
\hline Takes alcohol & $17(11.6)$ & $23(7.9)$ & $1.54(0.74-3.13)$ & 0.197 \\
\hline Does not take & $129(88.4)$ & $269(92.1)$ & 1 & \\
\hline \multicolumn{5}{|l|}{ Smoking } \\
\hline Smokes & $52(35.6)$ & $29(9.9)$ & $5.01(2.92-8.67)$ & $<0.001^{*}$ \\
\hline Do not smoke & $94(64.4)$ & $263(90.1)$ & 1 & \\
\hline \multicolumn{5}{|c|}{ Living with partner that smoked } \\
\hline Partner smokes & $68(46.6)$ & $80(27.4)$ & $2.31(1.49-3.56)$ & $<0.001^{*}$ \\
\hline Partner does not smoke & $78(53.4)$ & $212(72.6)$ & 1 & \\
\hline \multicolumn{5}{|c|}{ Herbal intake during pregnancy } \\
\hline Takes & $80(54.8)$ & $78(26.7)$ & $3.32(2.14-5.15)$ & $<0.001^{*}$ \\
\hline Not take & $66(45.2)$ & 214 (73.3) & 1 & \\
\hline
\end{tabular}

* p-value $<0.05$ is statistically significant; AoR (Adjusted odd Ratio)

consistent with findings of a population-based study in southern rural Ghana, which established a strong association between birth weight and maternal employment status. Mothers who were unemployed and coming from the poorest households were reported to be more likely to give birth to LBW babies compared to those who were gainfully employed [15]. Unemployment can contribute to poverty, leading to poor maternal nutritional intake [17]. According to Ahmed, et al., maternal undernutrition and inadequate dietary diversity during pregnancy are significant determinants of delivery of low birth weight babies [18].

The odds of delivering a LBW baby as observed in the current study, was higher among mothers who were anaemic during pregnancy $(\mathrm{Hb}<11.0 \mathrm{~g} / \mathrm{dl})$, compared to those who were not, similar to an earlier study also carried out in Ghana [12]. Other studies done in Ethiopia [7], Democratic Republic of Congo [17] and India [19] have also reported similar findings in which anaemia during pregnancy was found to be associated with LBW 
Table 5 Determinants of delivery of LBW babies, WAU, 2020

\begin{tabular}{|c|c|c|c|c|c|c|}
\hline Determinants & $\begin{array}{l}\text { Cases } \\
(n= \\
146)\end{array}$ & $\begin{array}{l}\text { Controls } \\
(n=292)\end{array}$ & $\begin{array}{l}\text { CoR } \\
(95 \% \mathrm{Cl})\end{array}$ & $p$-value & $\begin{array}{l}\text { AoR } \\
(95 \% \mathrm{Cl})\end{array}$ & $\begin{array}{l}p- \\
\text { value }\end{array}$ \\
\hline \multicolumn{7}{|l|}{ Mother's age (years) } \\
\hline$<20$ & 36 & 30 & $3.08(1.60-5.92)$ & $0.001^{*}$ & $1.75(0.33-9.25)$ & 0.509 \\
\hline $20-29$ & 82 & 190 & $1.10(0.66-1.84)$ & 0.688 & $1.11(0.52-2.37)$ & 0.778 \\
\hline$\geq 30$ & 28 & 72 & 1 & & 1 & \\
\hline \multicolumn{7}{|l|}{ Mother employment } \\
\hline Unemployed & 63 & 50 & $4.06(2.54-6.47)$ & $<0.001^{*}$ & $2.52(1.16-5.49)$ & $0.020^{*}$ \\
\hline Student & 20 & 39 & $1.65(0.89-3.03)$ & 0.106 & $2.67(0.93-7.64)$ & 0.066 \\
\hline Employed/self-employed & 63 & 203 & 1 & & 1 & \\
\hline \multicolumn{7}{|l|}{ Marital status } \\
\hline Single & 55 & 75 & $1.94(1.25-3.01)$ & $0.003^{*}$ & $1.35(0.58-3.16)$ & 0.487 \\
\hline Cohabiting/married husband unemployed & 15 & 15 & $2.65(1.23-5.69)$ & $0.012^{*}$ & $2.01(0.57-7.06)$ & 0.276 \\
\hline Cohabiting/married husband employed & 76 & 202 & 1 & & 1 & \\
\hline \multicolumn{7}{|l|}{ Mother's height (m) } \\
\hline$<1.5$ (short) & 47 & 52 & $2.19(1.34-3.55)$ & $0.001^{*}$ & $1.52(0.68-3.37)$ & 0.303 \\
\hline$\geq 1.5$ (normal) & 99 & 240 & 1 & & 1 & \\
\hline \multicolumn{7}{|l|}{ Gravidity } \\
\hline Primigravida(1) & 80 & 117 & $1.81(1.18-2.76)$ & $0.003^{*}$ & $1.77(0.80-3.90)$ & 0.152 \\
\hline Multigravida(> 1) & 66 & 175 & 1 & & 1 & \\
\hline \multicolumn{7}{|l|}{ ANC Visits } \\
\hline$<4$ times & 60 & 60 & $2.69(1.70-4.26)$ & $<0.001^{*}$ & $1.07(0.49-2.30)$ & 0.870 \\
\hline$\geq 4$ times & 86 & 232 & 1 & & 1 & \\
\hline \multicolumn{7}{|l|}{ Gestational age (weeks) } \\
\hline Preterm $(<37)$ & 134 & 213 & $12.58(1.66-94.84)$ & $0.014^{*}$ & $4.07(0.48-34.03)$ & 0.195 \\
\hline Term (37-40) & 11 & 59 & $3.72(0.45-30.72)$ & 0.221 & $2.60(0.27-25.45)$ & 0.410 \\
\hline Post term (> 40) & 1 & 20 & 1 & & 1 & \\
\hline
\end{tabular}

$1=$ Reference group; ${ }^{*}$ p-value $<0.05$ is statistically significant

delivery. Anaemia during pregnancy can limit maternal oxygen uptake, thus reducing oxygen supply to the foetus and thus contribute to foetal growth restriction [20].

In this study, babies born within less than 2 years after another child had higher odds of being of LBW, compared with those born after two or more years. Similar findings have been reported from Ethiopia [21, 22] and India [23]. This might be as a result of insufficient replacement of maternal nutrients used-up during the previous pregnancy and this may lead to reduced foetal development. A similar observation made in South Ethiopia by Mingude, et al. [16] has been explained on the basis that, the short inter-pregnancy interval is not able to allow the mother enough time to recover from the nutritional burden and stress of the previous pregnancy, resulting in maternal nutrition depletion. The short inter-pregnancy interval is also associated with maternal iron and folic acid depletion [18]. This reduces the ability of the mother to support foetal growth and development which in turn increases the possibility of growth restriction and LBW in subsequent pregnancies.

Our study also revealed that mothers who smoke cigarettes have higher odds of giving birth to a LBW baby. In a similar case-control study conducted in China [24], pregnant women who were exposed to even passive smoking had an increased risk of delivering low birth weight babies. Although, our study failed to determine the number of cigarette sticks smoked per day and for how long, other studies have reported that, mothers who are heavy smokers (> 8-10 cigarettes/day) had a higher odds of LBW babies [25]. Cigarette smoking is known to reduce oxygen supply to the foetus in-utero as carbon monoxide and nicotine-associated vasoconstriction reduces uterine and placental blood flow, thereby restricting the growth of the foetus, and hence can contribute to LBW [26]. Other factors including gravidity, maternal ill-health and taking of herbal medicine during 
Table 6 Determinants of delivery of LBW babies, WAU, 2020

\begin{tabular}{|c|c|c|c|c|c|c|}
\hline Determinants & $\begin{array}{l}\text { Cases } \\
(n=146)\end{array}$ & $\begin{array}{l}\text { Controls } \\
(n=292)\end{array}$ & $\begin{array}{l}\text { CoR } \\
(95 \% \mathrm{Cl})\end{array}$ & $p$-value & $\begin{array}{l}\text { AoR } \\
(95 \% \mathrm{Cl})\end{array}$ & $p$-value \\
\hline \multicolumn{7}{|c|}{ Mother anaemia status in pregnancy } \\
\hline Anaemic $(\mathrm{Hb}<11.0 \mathrm{~g} / \mathrm{dl})$ & 82 & 64 & $4.56(2.90-7.16)$ & $<0.001^{*}$ & $3.88(1.90-7.90)$ & $<0.001^{*}$ \\
\hline Not anaemic $(\mathrm{Hb} \geq 11.0 \mathrm{~g} / \mathrm{dl})$ & 64 & 228 & 1 & & 1 & \\
\hline \multicolumn{7}{|l|}{ Birth spacing } \\
\hline$<2$ years & 24 & 29 & $2.26(1.15-4.39)$ & $0.008^{*}$ & $2.53(1.11-5.73)$ & $0.026^{*}$ \\
\hline$\geq 2$ years & 58 & 159 & 1 & & 1 & \\
\hline \multicolumn{7}{|l|}{ Hypertension } \\
\hline Hypertensive & 47 & 28 & $4.47(2.57-7.83)$ & $<0.001^{*}$ & $1.45(0.63-3.36)$ & 0.387 \\
\hline Not hypertensive & 99 & 264 & 1 & & 1 & \\
\hline \multicolumn{7}{|l|}{ HIV } \\
\hline Positive & 11 & 7 & $3.31(1.14-10.29)$ & $0.010^{*}$ & $4.01(0.92-17.32)$ & 0.063 \\
\hline Negative & 135 & 285 & 1 & & 1 & \\
\hline \multicolumn{7}{|l|}{ Syphilis } \\
\hline Positive & 31 & 19 & $3.87(2.01-7.55)$ & $<0.001^{*}$ & $1.65(0.55-4.92)$ & 0.368 \\
\hline Negative & 115 & 273 & 1 & & 1 & \\
\hline \multicolumn{7}{|l|}{ Malaria } \\
\hline Positive & 106 & 137 & $2.99(1.91-4.73)$ & $<0.001^{*}$ & $1.80(0.88-3.65)$ & 0.103 \\
\hline Negative & 40 & 155 & 1 & & 1 & \\
\hline \multicolumn{7}{|l|}{ Iron \& folic acid used } \\
\hline$<3$ months & 34 & 23 & $3.55(1.92-6.60)$ & $<0.001^{*}$ & $1.04(0.39-2.77)$ & 0.933 \\
\hline$\geq 3$ months & 112 & 269 & 1 & & 1 & \\
\hline \multicolumn{7}{|l|}{ Smoking } \\
\hline Smokes & 52 & 29 & $5.01(2.92-8.67)$ & $<0.001^{*}$ & $4.36(1.94-9.80)$ & $<0.001^{*}$ \\
\hline Does not smoke & 94 & 263 & 1 & & 1 & \\
\hline \multicolumn{7}{|l|}{ Living with partner that smoked } \\
\hline Partner smokes & 68 & 80 & $2.31(1.49-3.56)$ & $<0.001^{*}$ & $0.91(0.45-1.84)$ & 0.798 \\
\hline Partner does not smoke & 78 & 212 & 1 & & 1 & \\
\hline \multicolumn{7}{|l|}{ Take herbal medicine } \\
\hline Takes & 80 & 78 & $3.32(2.14-5.15)$ & $<0.001^{*}$ & $1.92(0.99-3.72)$ & 0.051 \\
\hline Does not take & 66 & 214 & 1 & & 1 & \\
\hline
\end{tabular}

$1=$ Reference group; * $\mathrm{p}$-value $<0.05$ is statistically significant

pregnancy were not found to be significantly associated with the delivery of LBW babies in the current study after multivariable logistic regression analysis.

Even as our current study has identified four main maternal factors (unemployment, anaemia during pregnancy, short inter-pregnancy interval and smoking) as risk factors for the delivery of LBW babies in our study setting, Agbor et al. [4] found only one risk factor for LBW. In their review of eight years records of two health facilities in rural Cameroon, only preterm delivery was found to be associated LBW. Since a higher cut-off value $(2.7 \mathrm{~kg})$ was used to define LBW in their study, it was expected that more babies will be caught in the bracket of LBW (an additional 6.1\%) and possibly more risk factors identified. This difference in risk factors identified might be due to differences in the study settings. In an earlier study by Njim and colleagues also in Cameroon but in a sub-urban setting, many more risk factors were identified in addition to preterm delivery. Many factors are known to influence the duration of gestation and foetal growth, and thus, the birthweight [27]. These factors may relate to the mother or the physical environment and play an important role in determining the birthweight and the future health of the infant.

According to Johnson and colleagues [28] the risk factors for LBW are interrelated and that exposure to one factor increases the likelihood of exposure to a group of 
other factors which further increase the risk. Therefore, in addressing the issue of LBW, the risk factors should be tackled together using multi-component interventions. Our current study has identified two important modifiable risk factors for LBW ( $<2$ years interpregnancy interval and cigarette smoking during pregnancy) in addition to anaemia during pregnancy.

These three factors, can be adequately addressed through the implementation of recommendations already made by the WHO [13]. In order to achieve the goal of $30 \%$ reduction in the number of LBW infants between 2012 and 2025, WHO has recommended adequate nutrition for adolescent girls and the promotion of smoking cessation during and after pregnancy. Other recommendations include, intermittent iron and folic acid supplements for women of reproductive age and adolescent girls, prevention of malaria during pregnancy and adequate birth spacing.

In addition to iron and folic acid supplements, the issue of anaemia can be addressed through adequate nutrition using locally available foods, including fruits and vegetables. Education during antenatal sessions by attending midwives and dieticians can help in this direction. Prevention of malaria during pregnancy through the use insecticidal nets and intermittent preventive treatment with sulfadoxine-pyrimethamine (IPTp-SP) are also WHO recommendations to improve pregnancy outcomes in moderate to high malaria transmission areas in Africa.

Other interventions that will bring health care closer to the door-steps of pregnant women such as prenatal home-visitation that will focus on social support, health education and access to services, modelled similar to the community-based health planning and services (CHPS) concept in Ghana [29] can contribute to reducing LBW deliveries among at-risk women and adolescent girls in Sierra Leone. Using the CHPS concept, family planning can be promoted to widen the inter-pregnancy interval. Empowering women through job opportunities and formal education can also help reduce LBW, however these may take time to provide considering the current economic situation the world over and the peculiar Sierra Leonean situation where there is the need to recover economically from the 11 years civil war.

Although we employed a case-control study, which we consider an appropriate design for the study, the study is not without limitations as we did not match the cases and controls, which may introduce some selection bias. Assessment of some of the independent variables was liable to recall bias. The measurement we used for maternal smoking may not be the most suitable. Measuring the number of cigarettes sticks smoked by a mother in a day may better show the difference between the cases and controls. Also, the cut-off of $2.5 \mathrm{~kg}$ for LBW used in our assessment could exclude some babies from receiving critical clinical care available for LBW babies. These limitations notwithstanding, the study has identified important maternal factors that when addressed, can reduce the incidence of LBW deliveries in Sierra Leone and other developing countries with similar characteristics. The inclusion of all the referral facilities in the city strengthens our design as participants can better represent women of reproductive age in the catchment area of the health facilities.

\section{Conclusions}

This study has established that unemployment, anaemia during pregnancy, less than 2 years of inter-pregnancy interval, and cigarette smoking during pregnancy are significant factors for the delivery of LBW babies. There is a need for health care managers in WAU district to intensify screening and sensitization on the risk factors of LBW during antenatal sessions to address the identified factors among mothers. Also, the Ministry of Youth Affairs should design welfare programmes that would reduce the high rate of unemployment among women in Sierra Leone.

\begin{abstract}
Abbreviations
ANC: Antenatal care; AOR: Adjusted Odd Ratio; BMI: Body mass index; Cl: Confidence Interval; COR: Crude Odd Ratio; GFELTP: Ghana Field Epidemiology and Laboratory Training Programme; HIV: Human immunodeficiency virus; LBW: Low birth weight; NGO: Non-Governmental organization; PCMH: Princess Christian Maternity Hospital; SD: Standard deviation; SLDHS: Sierra Leone demographic health survey; SLESRC: Sierra Leone Ethics and Scientific Review Committee; SLFETP: Sierra Leone Field Epidemiology Training Programme; SPSS: Software package for social sciences; WAHO: West African Health Organization; WAU: Western Area Urban; WHO: World Health Organization
\end{abstract}

\section{Supplementary Information}

The online version contains supplementary material available at https://doi. org/10.1186/s13052-021-01160-y.

Additional file 1. Determinants of LBW in Western Area Urban District, Sierra Leone. Data on which findings of the study was based.

\section{Acknowledgements}

We recognize the technical support given by the staff of the Ghana Field Epidemiology and Laboratory Training Programme (GFELTP). We are also grateful to the West African Health Organization (WAHO) for financial support during the University program of the principal investigator. Our appreciation goes to the management of the Sierra Leone Field

Epidemiology Training Programme (SLFETP) for the technical support. Special thanks go to the Ministry of Health and Sanitation, hospital managements, study participants, and research assistants for their diverse contributions.

\section{Authors' contributions}

DKK, SS and FA, designed and conducted the study. DKK analyzed the data. DKK, SS, FA, KN, AA_L and EK were responsible for the interpretation of data. DKK drafted the manuscript, which all the authors critically reviewed and approved the final version.

Funding

This thesis work was supported by the University of Ghana and WAHO. 


\section{Availability of data and materials}

All data generated during the current study are included in this published article and its additional file.

\section{Declarations}

\section{Ethics approval and consent to participate}

This study was approved by the Sierra Leone Ethics and Scientific Review Committee (SLESRC). We obtained permission from the Chief Medical Officer, Ministry of Health and Sanitation (MoHS), and the hospital administration. Signed informed consent was obtained from each mother or her parent or guardian (mothers less than 16 years old) before data collection. The information collected was treated as confidential and codes were used to identify participants.

\section{Consent for publication}

Not applicable.

\section{Competing interests}

The authors affirmed that we have no conflicting interests.

\section{Author details}

${ }^{1}$ Field Epidemiology and Laboratory Training Programme, Accra, Ghana. ${ }^{2}$ Department of Epidemiology and Disease Control, School of Public Health, University of Ghana, Accra, Ghana. ${ }^{3}$ Field Epidemiology Training Program, Free Town, Sierra Leone.

\section{Received: 30 December 2020 Accepted: 24 September 2021} Published online: 28 October 2021

\section{References}

1. Tshotetsi L, Dzikiti L, Hajison P, Feresu S. Maternal factors contributing to low birth weight deliveries in Tshwane District, South Africa. PLoS ONE. 2019;14(3):1-13. https://doi.org/10.1371/journal.pone.0213058.

2. Hailu LD, Kebede DL. Determinants of low birth weight among deliveries at a referral Hospital in Northern Ethiopia. Biomed Res Int. 2018;2018:1-8. https://doi.org/10.1155/2018/8169615. Accessed 15 Dec 2020.

3. Muchemi OM, Echoka E, Makokha A. Factors associated with low birth weight among neonates born at Olkalou District Hospital, Central Region, Kenya. Pan Afr Med J. 2015;20:108. https://doi.org/10.11604/pamj.2015.20.1 08.4831 Accessed 23 Dec 2020.

4. Agbor VN, Ditah C, Tochie JN, Njim T. Low birthweight in rural Cameroon: an analysis of a cut-off value. BMC Pregnancy and Childbirth. 2018;18(1):30 https://doi.org/10.1186/s12884-018-1663-y. Accessed 29 Mar 2021.

5. Njim T, Atashili J, Mbu R, Choukem S-P. Low birth weight in a sub-urban area of Cameroon: an analysis of the clinical cut-off, incidence, predictors and complications. BMC Pregnancy and Childbirth. 2015;15(1):288. https:// doi.org/10.1186/s12884-015-0723-9. Accessed 29 Mar 2021.

6. Siyoum M, Melese T. Factors associated with low birth weight among babies born at Hawassa University Comprehensive Specialized Hospital, Hawassa, Ethiopia. Ital J Pediatr. 2019;45:48. https://doi.org/10.1186/s13052019-0637-7. Accessed 15 Dec 2020.

7. Gebrehawerya T, Gebreslasie K, Admasu E, Gebremedhin M. Determinants of low birth weight among mothers who gave birth in Debremarkos referral hospital, Debremarkos town, east Gojam, Amhara region. Ethiopia Neonatal Pediatr Med. 2018;04(01):1-6. Accessed 23 Dec 2020.

8. Statistics Sierra Leone (SSL) and ICF Maccro, Sierra Leone Demographic and Health Survey 2008; 2009.

9. Statistics Sierra Leone (SSL) and ICF International, Sierra Leone Demographic and Health Survey 2013; 2014.

10. Id LT, Dzikiti L, Hajison P, Id SF. Maternal factors contributing to low birth weight deliveries in Tshwane District. South Africa; 2019. https://doi.org/1 0.1371/journal.pone.0213058. Accessed 14 Dec 2020

11. Demelash H, Motbainor A, Nigatu D, Gashaw K, Melese A. Risk factors for low birth weight in Bale zone hospitals, South-East Ethiopia: a case control study. BMC Pregnancy Childbirth. 2015. https://doi.org/10.1186/s12 884-015-0677-y. Accessed 15 Dec 2020.

12. Adam Z, Ameme DK, Nortey P, Afari EA, Kenu E. Determinants of low birth weight in neonates born in three hospitals in Brong Ahafo region, Ghana, 2016- an unmatched case-control study. BMC Pregnancy Childbirth. 2019. https://doi.org/10.1186/s12884-019-2315-6. Accessed 15 Dec 2020.
13. WHO, WHA Global Nutrition Targets 2025: Low birth weight policy brief, World Health Organization, Geneva, 2014. https://wwwwhoint/nutrition/ topics/globaltargets_lowbirthweight_policybriefpdf. Accessed: 24 July 2021.

14. Alemu S, Workicho A, Nigatu M, Bokila T, Wolde T. Determinants of low birth weight in public health facilities, of Kambata Tembaro zone, South Ethiopia. PONS - Med Cas. 2018;15(2):66-74. https://doi.org/10.5937/pomc1 5-17839.

15. Manyeh AK, Kukula V, Odonkor G, Ekey RA, Adjei A, Narh-Bana S, et al. Socioeconomic and demographic determinants of birth weight in southern rural Ghana: evidence from Dodowa health and demographic surveillance system. BMC Pregnancy Childbirth. 2016;6(1):160. https://doi.org/10.1186/s12 884-016-0956-2. Accessed 15 Dec 2020.

16. Mingude AB, Gebretsadik W, Misker D, Woldeamanuel GG. Determinants of low birth weight among live birth newborns delivered at public hospitals in Gamo Gofa zone, South Ethiopia: unmatched case control study. SAGE Open Med. 2020;8:205031212094054. https://doi.org/10.1177/205031212094 0544. Accessed 15 Dec 2020.

17. Ilunga PM, Mukuku O, Mawaw PM, Mutombo AM, Lubala TK, Pongombo MSY, et al. Risk factors for low birth weight in Lubumbashi, Democratic Republic of the Congo. Med Sante Trop. 2016;26(4):386-90. https://doi.org/1 0.1684/mst.2016.0607.

18. Ahmed S, Hassen K, Wakayo T. A health facility based case-control study on determinants of low birth weight in Dassie town, Northeast Ethiopia: the role of nutritional factors. Nurt J. 2018;17(1):103. https://doi.org/10.1186/s12 937-018-0409-z. Accessed 15 Dec 2020.

19. Dubey DK, Nath DC. An epidemiological model investigating the association between mothers nutritional status and low birth weight in India. Health. 2016;08(03):03-261. https://doi.org/10.4236/health.2016.83027. Accessed 15 Dec 2020

20. Sharma SR, Giri S, Timalsina U, Bhandari SS, Basyal B, Wagle K, et al. Low birth weight at term and its determinants in a tertiary hospital of Nepal:a case-control study. PLoS ONE. 2015;10(4):4. https://doi.org/10.1371/journal. pone.0123962. Accessed 11 Dec 2020.

21. Hailemichael HT, Debelew GT, Alema HB, Weldu MG, Misgina KH. Determinants of adverse birth outcome in Tigrai region, North Ethiopia: Hospital-based case-control study. BMC Pediatr. 2020. https://doi.org/10.11 86/s12887-019-1835-6. Accessed 22 Dec 2020.

22. Endalamaw A, Engeda EH, Ekubagewargies DT, Belay GM, Tefera MA. Low birth weight and its associated factors in Ethiopia: a systematic review and meta-analysis. Ital J Pediatr. 2018;44(1):1. https://doi.org/10.1186/s13052-0180586-6. Accessed 15 Dec 2020.

23. Metgud CS, Naik VA, Mallapur MD. Factors affecting birth weight of a newborn - a community based study in rural Karnataka. India PLoS ONE. 2012;7(7):e40040. https://doi.org/10.1371/journal.pone.0040040. Accessed 15 Dec 2020.

24. Xi C, Luo M, Wang T, Wang Y, Wang S, Guo L, et al. Association between maternal lifestyle factors and low birth weight in preterm and term births: $A$ case-control study. Reprod Health. 2020;17:1. https://doi.org/10.1186/s12978020-00932-9 Accessed 20 Dec 2020.

25. Ko TJ, Tsai LY, Chu LC, Yeh SJ, Leung C, Chen CY, et al. Parental smoking during pregnancy and its association with low birth weight, small for gestational age, and preterm birth offspring: a birth cohort study. Pediatr Neonatol. 2014;55(1):1-27. https://doi.org/10.1016/j.pedneo.2013.05.005. Accessed 15 Nov 2020.

26. Andriani $\mathrm{H}, \mathrm{Kuo} \mathrm{H}-\mathrm{W}$. Adverse effects of parental smoking during pregnancy in urban and rural areas. BMC Pregnancy Childbirth. 2014;14:414. https://doi. org/10.1186/s12884-014-0414-y. Accessed 15 Dec 2020.

27. UNICEF, WHO. Low Birthweight: Country, regional and Global estimates. New York: UNICEF, Editorial and publications section; 2004. 1-27.

28. Johnson CD, Jones S, Paranjothy S. Reducing low birth weight: prioritizing action to address modifiable risk factors. J Public Health. 2017;39:122-31. https://doi.org/10.1093/pubmed/fdv212.

29. Nwameme AU, Tabong PTN, Adongo PB. Implementing community-based health planning and services in impoverished urban communities: health workers' perspective. BMC Health Serv Res. 2018;18(1):186. https://doi.org/1 0.1186/s12913-018-3005-1.

\section{Publisher's Note}

Springer Nature remains neutral with regard to jurisdictional claims in published maps and institutional affiliations. 